\title{
Finite element modeling of micropolar-based phononic crystals
}

\author{
Nicolás Guarín-Zapata*a, Juan Gomez ${ }^{\mathrm{a}}$, Camilo Valencia ${ }^{\mathrm{a}}$, Gary F. Dargush ${ }^{\mathrm{b}}$, Ali Reza \\ Hadjesfandiari $^{\mathrm{b}}$ \\ ${ }^{a}$ Universidad EAFIT, Departamento de Ingeniería Civil, Medellín, Colombia \\ Corresponding author: nguarinz@eafit.edu.co \\ ${ }^{b}$ Department of Mechanical and Aerospace Engineering, University at Buffalo
}

\begin{abstract}
The performance of a Cosserat/micropolar solid as a numerical vehicle to represent dispersive media is explored. The study is conducted using the finite element method with emphasis on Hermiticity, positive definiteness, principle of virtual work and Bloch-Floquet boundary conditions. The periodic boundary conditions are given for both translational and rotational degrees of freedom and for the associated force- and couple-traction vectors. Results in terms of band structures for different material cells and mechanical parameters are provided.
\end{abstract}

Keywords: cosserat media; micropolar elasticity; wave propagation; dispersive media; finite element method

\section{Introduction}

The increasing growth and strong development of the field of architectured materials during recent years has created a renewed interest in generalized or extended versions of classical continuum mechanics theories. Popular examples of these emerging disciplines can be identified in the area of phononic crystals and metamaterials $[1,2]$. These are materials which by virtue of their architectured microstructure exhibit unexpected mechanical properties at the macro level, such as negative refraction, negative bulk modulus or negative mass [3]. For example, the conversion from axial deformation into twist, would require chirality, which in turn would require an asymmetric stress tensor $[4,5]$. From the wave propagation perspective, these materials are attractive since they exhibit dispersive phenomena, such as filtering and directional effects over fixed frequency ranges [6]. Physically, such phenomena result from the interactions of the incident field with the microstructural elements producing local scattering and diffraction. Another wave phenomenon of 
interest is cloaking, where the propagation is directed around an object rendering it invisible. This is another application where there is a need for asymmetric stresses. For electromagnetic waves, this can be achieved through transformation optics [7].

The validation and effective use of these materials, particularly in dynamic analysis simulations involves two general steps. In the first place, it is necessary to conduct a design or characterization of the material in terms of its band structure or frequency-wave vector relation for a representative unit cell. This analysis step is typically conducted via Bloch analysis of the unit cell, which comprises solving a series of eigenvalue problems for a numerical model of the cell that explicitly includes all its microstructural elements. The solution from these analyses identify propagation frequencies associated to variations of the wave vector along the boundaries of the unit cell after considering the spatial periodicity of the material. The second analysis step involves the solution of a timedomain boundary value problem, which requires the consideration of a large number of material cells filling out the particular domain. Clearly, from a numerical point of view, the inclusion of the microstructural details in the numerical model implies prohibitive computational costs, thus requiring continuum based mechanical models with intrinsic capabilities to reproduce dispersive behavior.

Dispersion at the macroscopic level can be understood in terms of scattering and diffraction arising when the wave length of the free wave field approaches characteristic dimensions of the microstructural elements and, as a result, its treatment in terms of continuum mechanics requires theories involving constitutive length scale parameters. Broadly speaking, these class of models can be classified into (i) gradient based theories and (ii) enriched kinematics models. In the former, local kinematic descriptions retaining higher order displacement gradients are introduced resulting also in higher order stress tensors $[8,9,10,11,12,13,14,15,16,17,18,19]$. Alternatively, in the latter approach, the material point is endowed with additional degrees of freedom [20, 21, 16, 22].

This family of non-classical or generalized continuum models have been used in a wide range of applications in several research problems [23, 24, 25, 26, 27, 28, 29, 30, 31, 32, 33, 34]. However, there is a need to examine the strengths and weaknesses of the various models and to propose physical experiments that would be helpful in their critical assessment.

In this work we explore the capabilities of the micropolar model to capture dispersive behavior by virtue of its kinematic variables and particularly through the additional constitutive parameters. For that purpose, we address several theoretical and simulation aspects relevant to wave 
propagation in such micropolar medium. We start by reviewing the field equations for the model with special emphasis placed in the displacement-based equations of motion as these reveal phase velocities associated to the possible free wave modes. Following that section, we also discuss Bloch periodicity in the context of the micropolar theory. We show the relation between displacements and rotations, together with its corresponding traction components along the different regions of the unit cell properly accounting for the infinite character of the analysis domain in terms of a single material cell. Also, as will be shown later, the dispersion analysis via the Bloch theorem involves the solution of the frequency-domain reduced wave equation and thus it is useful to show the Hermitic and positive definite character of the boundary value problem. We show these two properties of the operator in section 2.1. The theoretical aspects of the paper also describe the variational statement and details for its finite element discretization, when conducting Bloch analysis. In the final part of the paper, we use the finite element formulation to test the capability of the micropolar model to capture dispersive behavior. First, and as a verification exercise of the formulation, we find the numerical and analytic band structure for a homogeneous micropolar continuum. The homogeneous cell analysis is also used to identify appropriate mesh properties in Bloch analysis of micropolar media. In a subsequent analysis aimed at producing further dispersion in the model, we also consider material cells with simple microstructures, namely a bilayer composite and a porous material composed of a circular cavity embedded in a micropolar matrix. These are simple microstructures, which facilitate the study of the variation of the dispersive properties by introducing changes in material and geometric parameters. In both cases, we find band structures for different values of the mechanical and geometric parameters and track the variation of the cut-off frequency associated to the microrotational waves.

\section{Micropolar Model}

The micropolar model used in this work introduces rotational mechanical interaction between

material points in terms of a couple-tractions vector $m_{i}^{(\hat{n})}$ defined through a generalized Cauchy's postulate as [35]:

$$
\lim _{\Delta S(\hat{n}) \rightarrow 0} \frac{\Delta M_{i}}{\Delta S(\hat{n})}=m_{i}^{(\hat{n})}
$$


where $\Delta S(\hat{n})$ is a small element of area oriented with $\hat{n}$ and $\Delta M_{i}$ is the resultant moment. The couple-tractions are completely described by the couple-stress tensor $\mu_{i j}$ according to

$$
m_{j}^{(\hat{n})}=\mu_{i j} \hat{n}_{i}
$$

In (1) above, $\Delta S(\hat{n})$ represents a surface material element with outward normal $\hat{n}$. Considering now the classical force per unit surface tractions vector $t_{j}^{(\hat{n})}$ related to the Cauchy stress tensor $\sigma_{i j}$, such as

$$
t_{j}^{(\hat{n})}=\sigma_{i j} \hat{n}_{i}
$$

leads to momentum and moment of momentum balance equations for the micropolar solid [22]:

$$
\begin{aligned}
& \sigma_{j i, j}+f_{i}=\rho \ddot{u}_{i} \\
& \sigma_{j k} \epsilon_{i j k}+\mu_{j i, j}+c_{i}=J \ddot{\theta}_{i}
\end{aligned}
$$

and where $f_{i}$ and $c_{i}$ are forces and moments per unit volume; $\rho$ and $J$ are the mass and rotational inertial densities, respectively, and $\epsilon_{i j k}$ is the Levi-Civita permutation tensor. In the model proposed by [18] the term $c_{i}$ is shown to be equivalent to a body force, while $J$ is eliminated at the onset. Here we retain the original form of the equations given in [22], where both terms are retained. Denoting displacements and microrotation vectors at a field point $\mathbf{x}$ and at the time instant $t$ by $u_{i}(\mathbf{x}, t)$ and $\theta_{i}(\mathbf{x}, t)$, respectively, we have that the local deformation $\gamma_{j i}$ at the material point is now the difference between the displacement gradients $u_{i, j}$ and the microrotation (vector) $\theta_{k}$

$$
\gamma_{j i}=u_{i, j}-\epsilon_{k j i} \theta_{k}
$$

Also, notice that the consideration of the independent microrotational field introduces an additional kinematic variable in the form of a generalized curvature-twist $\kappa_{j i}$ describing the change of microrotation per unit length

$$
\kappa_{j i}=\theta_{i, j}
$$

In a linear isotropic elastic micropolar medium the constitutive equations take the following form [22]:

$$
\begin{aligned}
\sigma_{j i} & =(\mu+\alpha) \gamma_{j i}+(\mu-\alpha) \gamma_{i j}+\lambda \gamma_{k k} \delta_{i j}, \\
\mu_{j i} & =(\eta+\varepsilon) \kappa_{j i}+(\eta-\varepsilon) \kappa_{i j}+\beta \kappa_{k k} \delta_{i j} .
\end{aligned}
$$


where $\mu$ and $\lambda$ are the known Lamé parameters from classical elasticity, while $\alpha, \beta, \eta$ and $\varepsilon$ are extra material parameters from the micropolar model and representative of additional particle interactions. The set of constitutive equations can also be written in the following alternative form:

$$
\begin{aligned}
& \sigma_{j i}=\mu \gamma_{i j}^{S}+2 \alpha \gamma_{i j}^{A}+\lambda \gamma_{k k} \delta_{i j}, \\
& \mu_{j i}=\eta \kappa_{i j}^{S}+2 \varepsilon \kappa_{i j}^{A}+\beta \kappa_{k k} \delta_{i j},
\end{aligned}
$$

where the superscripts $S$ and $A$ denote the symmetric and skew-symmetric parts of the associated second order tensors. We can mention that $\beta$ is a parameter only related to torsion, using a parallel with classical elasticity it resembles the role of $\lambda$. On the other hand, $\eta$ is related to torsion and bending while $\varepsilon$ is only related to bending modulus. Furthermore, $\alpha$ is known as micropolar couple modulus and quantifies the coupling between micro and macrorotation — see [36, 37, 38] for further discussion on the interpretation of these parameters.

Using (7) together with (5) and (6) in the linear and angular momentum balance equations leads to displacement time-domain equations of motion:

$$
\begin{array}{r}
(\lambda+2 \mu) u_{k, k i}-\epsilon_{i j k} \epsilon_{k l m}(\mu+\alpha) u_{m, l j}+2 \alpha \epsilon_{i j k} \theta_{k, j}+f_{i}=\rho \ddot{u}_{i} \\
(\beta+2 \eta) \theta_{k, k i}-\epsilon_{i j k} \epsilon_{k l m}(\eta+\varepsilon) \theta_{m, l j}+2 \alpha \epsilon_{i j k} u_{k, j}-4 \alpha \theta_{i}+c_{i}=J \ddot{\theta}_{i} .
\end{array}
$$

With boldface characters denoting vector fields, the equations of motion can also be written for completeness in explicit form as:

$$
\begin{aligned}
& (\lambda+2 \mu) \boldsymbol{\nabla} \boldsymbol{\nabla} \cdot \mathbf{u}-(\mu+\alpha) \boldsymbol{\nabla} \times \boldsymbol{\nabla} \times \mathbf{u}+2 \alpha \boldsymbol{\nabla} \times \boldsymbol{\theta}+\mathbf{f}=\rho \frac{\partial^{2} \mathbf{u}}{\partial t^{2}}, \\
& (\beta+2 \eta) \boldsymbol{\nabla} \boldsymbol{\nabla} \cdot \boldsymbol{\theta}-(\eta+\varepsilon) \boldsymbol{\nabla} \times \boldsymbol{\nabla} \times \boldsymbol{\theta}+2 \alpha \boldsymbol{\nabla} \times \mathbf{u}-4 \alpha \boldsymbol{\theta}+\mathbf{c}=J \frac{\partial^{2} \boldsymbol{\theta}}{\partial t^{2}} .
\end{aligned}
$$

When considering plane problems the term $\boldsymbol{\nabla} \boldsymbol{\nabla} \cdot \boldsymbol{\theta}$ is zero and the behavior will not depend on the parameter $\beta$, see Appendix $\mathrm{B}$ for the explicit expressions for plane problems.

Notice in $(8 \mathrm{~b})$ and $(9 \mathrm{~b})$ the appearance of the dilatation $\boldsymbol{\nabla} \cdot \boldsymbol{\theta}$, which implicitly assumes that $\boldsymbol{\theta}$ is not, in fact, a pure rotation (or microrotation). On the other hand, if $\boldsymbol{\nabla} \cdot \boldsymbol{\theta}=0$ is enforced, then the resulting theory would suffer from the same type of indeterminacy as the original couple stress theory [11]. Following the current convention, however, $\boldsymbol{\theta}$ will continue to be called the microrotation in this paper.

In order to conduct Bloch analysis, and more specifically to determine the dispersion relations for a micropolar solid, it is convenient to neglect the body force and couple densities and to assume 
a time dependence of the form $e^{-i \omega t}$ for both the displacement and rotation field, therefore yielding the following reduced frequency-domain version of the equations of motion:

$$
\begin{array}{r}
(\lambda+2 \mu) \boldsymbol{\nabla} \boldsymbol{\nabla} \cdot \mathbf{u}-(\mu+\alpha) \boldsymbol{\nabla} \times \boldsymbol{\nabla} \times \mathbf{u}+2 \alpha \boldsymbol{\nabla} \times \boldsymbol{\theta}=-\rho \omega^{2} \mathbf{u}, \\
(\beta+2 \eta) \boldsymbol{\nabla} \boldsymbol{\nabla} \cdot \boldsymbol{\theta}-(\eta+\varepsilon) \boldsymbol{\nabla} \times \boldsymbol{\nabla} \times \boldsymbol{\theta}+2 \alpha \boldsymbol{\nabla} \times \mathbf{u}-4 \alpha \boldsymbol{\theta}=-J \omega^{2} \boldsymbol{\theta} .
\end{array}
$$

For convenience in later developments and to aid the comparison with similar formulations available in the literature, it is convenient to use also the alternative form:

$$
\begin{aligned}
c_{1}^{2} \nabla \boldsymbol{\nabla} \cdot \mathbf{u}-c_{2}^{2} \boldsymbol{\nabla} \times \boldsymbol{\nabla} \times \mathbf{u}+K^{2} \boldsymbol{\nabla} \times \boldsymbol{\theta} & =-\omega^{2} \mathbf{u}, \\
c_{3}^{2} \nabla \boldsymbol{\nabla} \cdot \boldsymbol{\theta}-c_{4}^{2} \boldsymbol{\nabla} \times \boldsymbol{\nabla} \times \boldsymbol{\theta}+Q^{2} \boldsymbol{\nabla} \times \mathbf{u}-2 Q^{2} \boldsymbol{\theta} & =-\omega^{2} \boldsymbol{\theta}
\end{aligned}
$$

and where: $c_{1}$ represents the phase/group speed for the longitudinal wave $(P)$ that is non-dispersive as in the classical case, $c_{2}$ represents the high-frequency limit $(k \rightarrow \infty)$ phase/group speed for a transverse wave $(S)$ that is dispersive unlike the classical counterpart, $c_{3}$ represents the highfrequency limit $(k \rightarrow \infty)$ phase/group speed for a longitudinal-rotational wave $(L R)$ with a corkscrew-like motion that is dispersive and does not have a classical counterpart, $c_{4}$ represents the high-frequency limit $(k \rightarrow \infty)$ phase/group speed for a transverse-rotational wave $(T R)$ that is dispersive and does not have a classical counterpart, $Q$ represents the cut-off frequency for rotational waves appearance, and $K$ quantifies the difference between the low-frequency and high-frequency phase/group speed for the S-wave - see fig. 1 for a qualitative description. These parameters are defined by:

$$
\begin{array}{cc}
c_{1}^{2}=\frac{\lambda+2 \mu}{\rho}, & c_{3}^{2}=\frac{\beta+2 \eta}{J}, \\
c_{2}^{2}=\frac{\mu+\alpha}{\rho}, & c_{4}^{2}=\frac{\eta+\varepsilon}{J}, \\
Q^{2}=\frac{2 \alpha}{J}, & K^{2}=\frac{2 \alpha}{\rho} .
\end{array}
$$

We should highlight that $c_{3}$ does not play a role for waves in the plane. As mentioned before, $\boldsymbol{\nabla} \boldsymbol{\nabla} \cdot \boldsymbol{\theta}=0$ in that case.

The Principle of Virtual Work (PVW) for a micropolar solid follows after considering the translational and rotational equilibrium equations, using the virtual fields $\delta u_{i}$ and $\delta \theta_{i}$ as weighting functions and integrating over the volume $V$ with the aid of the divergence theorem. Denoting the kinematic measures in a micropolar solid by $\gamma_{i j}^{S}, \gamma_{i j}^{A}$ and $\kappa_{i j}$ corresponding to the classical infinitesimal strain tensor, the skew-symmetric part of the relative deformation tensor - i.e., the difference 
between the displacement gradient and the micro-displacement gradient — and the generalized curvature-twist tensor allow us to write this principle in the form

$$
\begin{array}{r}
\int_{V} \sigma_{i j}^{S} \delta \gamma_{i j}^{S} \mathrm{~d} V+\int_{V} \sigma_{i j}^{A} \delta \gamma_{i j}^{A} \mathrm{~d} V+\int_{V} \mu_{i j} \delta \kappa_{i j} \mathrm{~d} V-\int_{S} t_{i} \delta_{i} \mathrm{~d} S-\int_{S} m_{i} \delta \theta_{i} \mathrm{~d} S- \\
\omega^{2} \int_{V} \rho u_{i} \delta u_{i} \mathrm{~d} V-\omega^{2} \int_{V} J \theta_{i} \delta \theta_{i} \mathrm{~d} V=0
\end{array}
$$

where $\sigma_{i j}^{S}$ is the symmetric part of the stress tensor, while $\sigma_{i j}^{A}$ is the skew-symmetric part of the stress tensor.

\section{Formulation for Periodic Materials}

Here we review the relevant aspects of the analysis of spatially periodic materials in terms of the theory of phononic crystals, and particularly the so-called Bloch-Floquet periodic boundary conditions applied to the micropolar solid. For an in-depth discussion of periodic materials the reader is referred to classical sources [39, 40], while a comprehensive review is provided in [1]. In that theory, the key concept is established by Bloch's theorem, providing a relationship between the fields on opposite sides of the cell and taking into account spatial periodicity in a wave propagation problem. Therefore, the characterization of the micropolar medium is to be conducted after assuming that the material is the result of the spatial and periodic repetition of a fundamental unit cell. Under this assumption, a fundamental cell containing a motif repeats itself (in one, two, or three space dimensions) according to a spatial period defined in terms of a lattice vector. The motif refers to a microstructural heterogeneity which could contain different materials and geometries, as well as fluids and/or solids of the classical or Cosserat type. The dispersive properties of such a periodic material, given in terms of frequency-wave number relations (or band diagram), can be found from the analysis of a single fundamental cell after using Bloch's theorem, which establishes that a function $\mathbf{u}(\mathbf{x})$ can be expressed in the form

$$
\mathbf{u}(\mathbf{x})=\mathbf{w}(\mathbf{x}) e^{i \mathbf{k} \cdot \mathbf{x}}
$$

where $\mathbf{w}(\mathbf{x})$ is the Bloch function that has the same periodicity of the material and $\mathbf{k}$ is a wave vector. Accordingly, the solution is the product of a periodic function with the periodicity of the lattice and a plane wave, that is also periodic. As a consequence, the field variables in the differential 
equation satisfy the relation

$$
\mathbf{\Phi}(\mathbf{x}+\mathbf{a})=\mathbf{\Phi}(\mathbf{x}) e^{i \mathbf{k} \cdot \mathbf{a}}
$$

connecting the variable $\boldsymbol{\Phi}$ at opposite sides of the unit cell set apart by a vector a. In this case, $\boldsymbol{\Phi}$ refers to the principal variable (or any of its spatial derivatives) involved in the physical problem. It then follows that if one wants to characterize the material in terms of its wave propagation velocities to obtain values that can be used in a homogenized continuum model, it suffices to analyze a single cell. In the case of the micropolar medium, Bloch's theorem states that the eigenfunctions of (10) can be expressed in the form

$$
\begin{aligned}
& u_{r}(\mathbf{x})=u_{r}(\mathbf{x}+\mathbf{a}) e^{i \mathbf{k} \cdot \mathbf{a}} \\
& \theta_{r}(\mathbf{x})=\theta_{r}(\mathbf{x}+\mathbf{a}) e^{i \mathbf{k} \cdot \mathbf{a}}
\end{aligned}
$$

where $\mathbf{a}$ is a vector that represents the periodicity of the material. That is, the solution is the same at opposite sides of the unit cell, except for a phase shift factor $e^{i \mathbf{k} \cdot \mathbf{a}}$. Due to the linearity of the differential equations we also have the following Bloch-periodic boundary conditions for the corresponding traction vectors

$$
\begin{aligned}
& t_{r}(\mathbf{x})=-t_{r}(\mathbf{x}+\mathbf{a}) e^{i \mathbf{k} \cdot \mathbf{a}} \\
& m_{r}(\mathbf{x})=-m_{r}(\mathbf{x}+\mathbf{a}) e^{i \mathbf{k} \cdot \mathbf{a}}
\end{aligned}
$$

Thus, in the case of the micropolar solid, Bloch's theorem reduces to the following set of boundary conditions for displacements, microrotations, force-tractions and couple-tractions:

$$
\begin{aligned}
& u_{r}(\mathbf{x})=u_{r}(\mathbf{x}+\mathbf{a}) e^{i \mathbf{k} \cdot \mathbf{a}} \\
& \theta_{r}(\mathbf{x})=\theta_{r}(\mathbf{x}+\mathbf{a}) e^{i \mathbf{k} \cdot \mathbf{a}} \\
& t_{r}(\mathbf{x})=-t_{r}(\mathbf{x}+\mathbf{a}) e^{i \mathbf{k} \cdot \mathbf{a}} \\
& m_{r}(\mathbf{x})=-m_{r}(\mathbf{x}+\mathbf{a}) e^{i \mathbf{k} \cdot \mathbf{a}}
\end{aligned}
$$

The set of conditions summarized in (14) will be satisfied in a variational sense using a finite element formulation. Subsequently, a numerical model of the unit cell resulting in a generalized eigenvalue problem will be solved for various specifications of the wave vector. The details of such an implementation will be discussed next. 


\subsection{Hermiticity of the equations}

Since the dynamic analysis of the micropolar medium involves solution of the frequency domain reduced wave equation subject to Bloch periodic boundary conditions, as given by (14), it becomes necessary to rewrite the PVW (12) so it can be used to properly represent inner products in complex-valued vector spaces. Using the operator $*$ to denote complex conjugate, we can write:

$$
\begin{aligned}
\delta \Pi\left(\omega, u_{i}, \hat{u}_{i}\right)= & \int_{V} \tau_{i j}^{*} \hat{\epsilon}_{i j} \mathrm{~d} V+\int_{V} \sigma_{i j}^{*} \hat{\gamma}_{i j}^{A} \mathrm{~d} V+\int_{V} \mu_{i j}^{*} \hat{\kappa}_{i j} \mathrm{~d} V-\int_{S} t_{i}^{*} \hat{u}_{i} \mathrm{~d} S \\
& -\int_{S} m_{i}^{*} \hat{\theta}_{i} \mathrm{~d} S-\omega^{2} \int_{V} \rho u_{i}^{*} \hat{u}_{i} \mathrm{~d} V-\omega^{2} \int_{V} J \theta_{i}^{*} \hat{\theta}_{i} \mathrm{~d} V \equiv 0 .
\end{aligned}
$$

In the principle of virtual work given by (15) $u_{i}$ and $\hat{u}_{i}$ represent the actual and virtual fields respectively. In the sense of (15), $\delta \Pi$ could be understood as the variation of the Lagrangian functional of the micropolar system. As will be demonstrated next, if we interchange the variables $u_{i}$ and $\hat{u}_{i}$, we find that

$$
\delta \Pi\left(\omega, u_{i}, \hat{u}_{i}\right)=\delta \Pi\left(\omega, \hat{u}_{i}, u_{i}\right)
$$

which implies that the operator is Hermitian (self-adjoint) under Bloch periodic boundary conditions also resulting in Hermitian matrices when discretized via finite elements.

The proof of Hermiticity follows after one uses Bloch periodicity conditions between the tractions and displacements (see (14)) into the boundary terms in (15), which yields

$$
\begin{aligned}
& \int_{S} t_{r}^{*}(\mathbf{x}) u_{r}(\mathbf{x}) \mathrm{d} S+\int_{S} m_{r}^{*}(\mathbf{x}) \theta_{r}(\mathbf{x}) \mathrm{d} S= \\
& \sum_{p}\left\{\int_{S_{p}}\left[t_{r}^{*}(\mathbf{x}) u_{r}(\mathbf{x})+t_{r}^{*}\left(\mathbf{x}+\mathbf{a}_{p}\right) u_{r}\left(\mathbf{x}+\mathbf{a}_{p}\right)\right] \mathrm{d} S_{p}+\right. \\
& \left.\int_{S_{p}}\left[m_{r}^{*}(\mathbf{x}) \theta_{r}(\mathbf{x})+m_{r}^{*}\left(\mathbf{x}+\mathbf{a}_{p}\right) \theta_{r}\left(\mathbf{x}+\mathbf{a}_{p}\right)\right] \mathrm{d} S_{p}\right\},
\end{aligned}
$$

where the index $p$ refers to each pair of opposite sides of the boundary. Introducing the phase shifts 
and elaborating further gives:

$$
\begin{aligned}
& \int_{S} t_{r}^{*}(\mathbf{x}) u_{r}(\mathbf{x}) \mathrm{d} S+\int_{S} m_{r}^{*}(\mathbf{x}) \theta_{r}(\mathbf{x}) \mathrm{d} S= \\
& \sum_{p}\left\{\int_{S_{p}} u_{r}(\mathbf{x})\left[t_{r}^{*}(\mathbf{x})+e^{-i \mathbf{k} \cdot \mathbf{a}} t_{r}^{*}\left(\mathbf{x}+\mathbf{a}_{p}\right)\right] \mathrm{d} S_{p}+\right. \\
& \left.\int_{S_{p}} \theta_{r}(\mathbf{x})\left[m_{r}^{*}(\mathbf{x})+e^{-i \mathbf{k} \cdot \mathbf{a}} m_{r}^{*}\left(\mathbf{x}+\mathbf{a}_{p}\right)\right] \mathrm{d} S_{p}\right\}
\end{aligned}
$$

which after taking the complex conjugate reduces to the Bloch-equilibrium condition (or the relationship between the traction vectors at opposite faces of the cell) for the terms enclosed by the square brackets. This leads to the vanishing of the boundary terms proving the Hermiticity condition.

\subsection{Positive definiteness}

To demonstrate positive (semi)-definiteness it is convenient to define the total potential and kinetic energy functionals $U([u, \theta],[u, \theta])$ and $T([u, \theta],[u, \theta])$ such that

$$
\begin{aligned}
& U([u, \theta],[u, \theta])=\int_{V} \gamma_{i j}^{S *} C_{i j k l} \gamma_{i j}^{S} \mathrm{~d} V+\int_{V} \alpha \gamma_{i j}^{A *} \gamma_{i j}^{A} \mathrm{~d} V+\int_{V} \kappa_{i j}^{*} D_{i j k l} \kappa_{i j} \mathrm{~d} V \\
& T([u, \theta],[u, \theta])=\int_{V} \rho u_{i}^{*} u_{i} \mathrm{~d} V+\int_{V} J \theta_{i}^{*} \theta_{i} \mathrm{~d} V .
\end{aligned}
$$

For the potential functional to be positive definite we need that the constitutive tensors are positive definite. For an isotropic material, this implies the following constraints in the material parameters:

$$
\begin{aligned}
& \mu>0, \quad \alpha>0, \quad \eta>0, \quad \varepsilon>0, \\
& 3 \lambda+2 \mu>0, \quad 3 \beta+2 \eta>0 .
\end{aligned}
$$

It should be noted that there exist differences in notation in the literature for material parameters. Particularly, the symbols used by [16] are known to be confusing since the symbol $\mu$ is used for a combination of the classic Lamé parameter and a micropolar parameter. The use of this symbol have led to incorrect inequalities, as presented by [41] and [37]. 
In order to have the condition $[u, \theta] \neq 0$, the functional $T$ should be different from zero implying that

$$
\omega_{r}^{2}=\frac{U\left(\left[u_{r}, \theta_{r}\right],\left[u_{r}, \theta_{r}\right]\right)}{T\left(\left[u_{r}, \theta_{r}\right],\left[u_{r}, \theta_{r}\right]\right)},
$$

meaning that $\omega$ is always greater than or equal to zero. This result is in agreement with the physical meaning of angular frequency given to $\omega$. On the other hand, the potential energy could be zero in the case of rigid body motion implying that the form $U$ is positive semi-definite, while the form $T$ is positive definite and so are their discrete counterparts.

\section{FEM formulation}

Finite element equations for the micropolar solid are straightforward to obtain after introducing the displacement and microrotation interpolation functions ${ }_{u} N_{i}^{k}$ and ${ }_{\theta} N_{i}^{k}$. Here the superscript $k$ makes reference to the contribution from the $k$ th node of a given element, while the right subscript $i$ indicates the tensorial nature of the variable being interpolated. The displacement and microrotation vectors at a given point inside the element can now be written in terms of the nodal variables $u^{k}$ and $\theta^{k}$ as

$$
u_{i}={ }_{u} N_{i}^{k} u^{k}, \quad \theta_{i}={ }_{\theta} N_{i}^{k} \theta^{k} .
$$

where the summation convention for repeated indices applies for both physical and interpolation subscripts and superscripts. For the actual numerical implementation, it is convenient to express the microrotation vector $\theta_{i}$ in terms of its dual skew-symmetric rotation tensor $\theta_{i j}$, such that

$$
\theta_{i j}=\epsilon_{q i j}{ }_{\theta} N_{q}^{k} \theta^{k} \equiv R_{i j}^{k} \theta^{k}
$$

Kinematic descriptions in terms of derivatives of the primary fields $u_{i}$ and $\theta_{i}$ follow from suitable combinations of derivatives of the primary interpolation functions. Thus,

$$
\begin{array}{r}
\gamma_{i j}^{S}=B_{i j}^{k} u^{k}, \quad \omega_{i j}=\hat{W}_{i j}^{k} u^{k}, \\
\kappa_{i j}=M_{i j}^{k} \theta^{k}, \quad \gamma_{i j}^{A}=\hat{W}_{i j}^{k} u^{k}+R_{i j}^{k} \theta^{k} .
\end{array}
$$

where the tensor $\omega_{i j}$ denotes the skew-symmetric part of the displacement gradient. Substitution 
of (19) and (20) into (12) yields the PVW in terms of virtual nodal variables $\delta u^{k}$ and $\delta \theta^{k}$

$$
\begin{array}{r}
\delta u^{k} \int_{v} B_{i j}^{k} \sigma_{i j}^{S} \mathrm{~d} V+\delta u^{k} \int_{V} \hat{W}_{i j}^{k} \sigma_{i j}^{A} \mathrm{~d} V+\delta \theta^{k} \int_{V} R_{i j}^{k} \sigma_{i j}^{A} \mathrm{~d} V \\
+\delta \theta^{k} \int_{V} M_{i j}^{k} \mu_{i j} \mathrm{~d} V-\omega^{2} \delta u^{k} \int_{V} \rho_{u} N_{i}^{k} u_{i} \mathrm{~d} V-\omega^{2} \delta \theta^{k} \int_{V} J{ }_{\theta} N_{i}^{k} \theta_{i} \mathrm{~d} V \\
-\delta u^{k} \int_{S}{ }_{u} N_{i}^{k} t_{i} \mathrm{~d} S-\delta \theta^{k} \int_{S}{ }_{\theta} N_{i}^{k} m_{i} \mathrm{~d} S=0 .
\end{array}
$$

Using the arbitrary character of the virtual fundamental fields $\delta u^{k}$ and $\delta \theta^{k}$ in (21), gives the following set of weak equilibrium equations in terms of nodal forces and couples consistent with the stresses and couple stresses;

$$
\begin{array}{r}
\hat{f}_{\tau}^{k}+\hat{f}_{\sigma}^{k}-\hat{f}_{I}^{k}-\hat{T}^{k}=0, \\
\hat{m}_{\sigma}^{k}+\hat{m}_{\mu}^{k}-\hat{m}_{I}^{k}-\hat{q}^{k}=0 .
\end{array}
$$

where the different terms become obvious after comparing (21) and (22). These equations describing equilibrium of forces and moments for the $k$-th degree of freedom can be written in the following matrix form

$$
\left[\begin{array}{cc}
K_{u u}^{k p} & K_{u \theta}^{k p} \\
K_{\theta u}^{k p} & K_{\theta \theta}^{k p}
\end{array}\right]\left\{\begin{array}{l}
u^{p} \\
\theta^{p}
\end{array}\right\}-\omega^{2}\left[\begin{array}{cc}
M_{u u}^{k p} & 0 \\
0 & M_{\theta \theta}^{k p}
\end{array}\right]\left\{\begin{array}{l}
u^{p} \\
\theta^{p}
\end{array}\right\}=\left\{\begin{array}{l}
f_{\text {ext }} \\
q_{\text {ext }}
\end{array}\right\},
$$

which results after writing the stress-strain relationships for the micropolar solid in terms of constitutive tensors $C_{i j k l}, G_{i j k l}$ and $D_{i j k l}$ as

$$
\begin{aligned}
& \sigma_{i j}=C_{i j k l} \gamma_{k l}^{S}+G_{i j k l} \gamma_{k l}^{A}, \\
& \mu_{i j}=D_{i j k l} \kappa_{k l} .
\end{aligned}
$$

For completeness, all of the terms in the matrix equation for the micropolar solid are given in Appendix A.

Imposition of the Bloch periodic boundary conditions in the system given by (22) results in a generalized eigenvalue problem of the form [42]:

$$
\left[K_{R}-\omega^{2} M_{R}\right]\left\{\mathbf{U}_{R}\right\}=\mathbf{0} .
$$

\section{Dispersion relations for a micropolar cellular material}

We now conduct a series of numerical simulations intended to test the capabilities of the micropolar model as a numerical vehicle to introduce dispersive behavior through a continuum based 
approach. For that purpose, we consider first the simplest case of a homogeneous material cell. This ideal case is also useful as a verification problem for the numerical implementation as that model has a closed-form dispersion relation. At the same time, the homogeneous case is used to assess the convergence of the band structure predicted by the numerical model. The particularization of the equations of motion (8) to the in-plane 2D problem is described in Appendix B. In a subsequent analysis, and intended to identify the sensitivity of the micropolar material parameters in the band structure, we also performed Bloch analysis for a bilayer composite. In this case, the band structures were found for different values of a single mechanical parameter, while keeping constant values for the remaining ones. As a final test, we extended our analysis to a cellular material with a microstructure corresponding to a circular pore embedded in an otherwise micropolar medium. In this case, we wanted to test the sensitivity of the dispersive response to the relative size of the pore with respect to the length scale constitutive parameter implicit in the material model.

\subsection{Homogeneous material}

In a homogeneous micropolar solid, dispersion relations can be obtained in closed-form (See fig. 1). Following, we present the expressions for a two-dimensional solid. The frequency-wavenumber relationships can be written in compact form as

$$
\begin{aligned}
& \omega_{m, n}^{P}=c_{1} k_{m, n}, \\
& \omega_{m, n}^{S}=\sqrt{\frac{A}{2}-\frac{1}{2} \sqrt{A^{2}-4 B}}, \\
& \omega_{m, n}^{T R}=\sqrt{\frac{A}{2}+\frac{1}{2} \sqrt{A^{2}-4 B}},
\end{aligned}
$$

where the constants $A$ and $B$ correspond to

$$
\begin{aligned}
& A=2 Q^{2}+\left(c_{2}^{2}+c_{4}^{2}\right) k_{m, n}^{2} \\
& B=2 Q^{2} c_{2}^{2} k_{m, n}^{2}-K^{2} Q^{2} k_{m, n}^{2}+c_{2}^{2} c_{4}^{2} k_{m, n}^{4}
\end{aligned}
$$

and TR refers to transverse-rotational.

One difference between wave propagation in micropolar and classical elasticity is the appearance of new (rotational) propagating waves that are dispersive. These waves appear above a cut-off

frequency given by $\omega_{0}^{2}=2 Q^{2}$ [22]. Besides the two propagation modes mentioned above there is another one with the following frequency-wavenumber relation

$$
\omega_{m, n}^{L R}=\sqrt{2 Q^{2}+c_{3}^{2} k_{m, n}^{2}},
$$


where LR refers to longitudinal-rotational. This wave only exists in 3D, and will not be present in the following results.

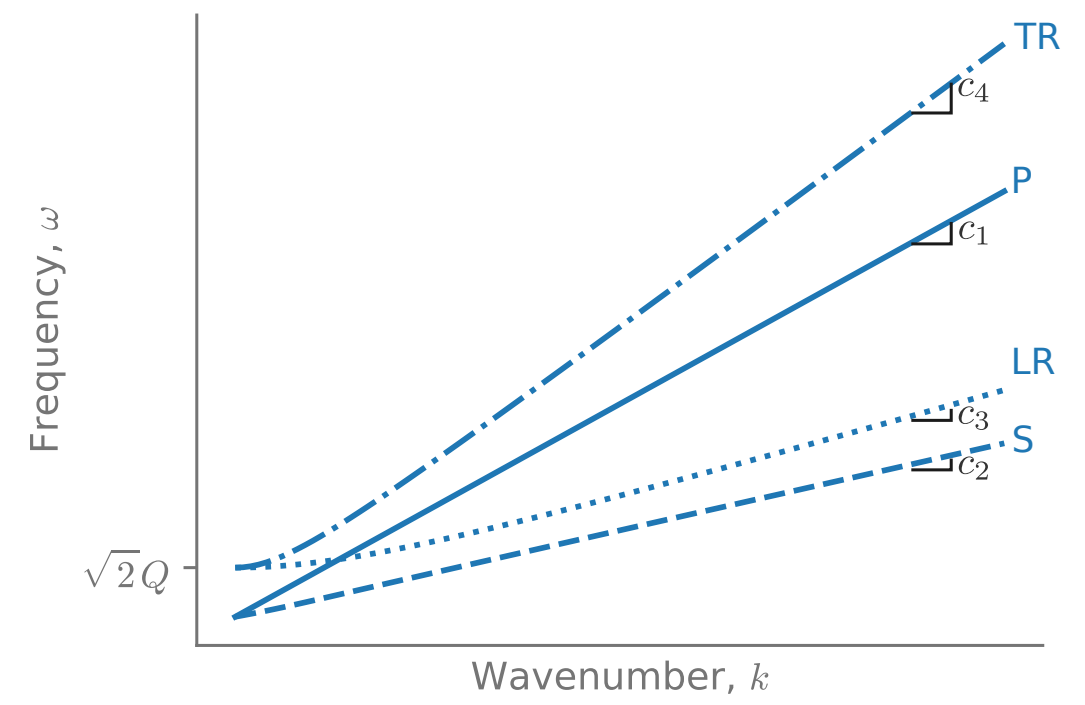

Figure 1. Qualitative depiction of the dispersion relations for a micropolar material. The different branches are: $(P)$ non-dispersive longitudinal wave with phase/group speed $c_{1}$ as in the classical case, $(S)$ dispersive transverse wave with limiting $(k \rightarrow \infty)$ phase/group speed $c_{2},(L R)$ dispersive longitudinalrotational (corkscrew-like) wave with limiting $(k \rightarrow \infty)$ phase/group speed $c_{3}$, (TR) dispersive transverserotational wave wave with limiting $(k \rightarrow \infty)$ phase/group speed $c_{4}$.

When conducting Bloch analysis for a 2D cell, the Bloch theorem requires that the wave number vector is swept over the first Brillouin zone [39]. As a result, the $m$ and $n$ subscripts in the frequency and wave number terms represent values of the wave number along adjacent Brillouin zones and refer to waves coming from these adjacent Brillouin zones. These wave numbers are given by:

$$
k_{m, n}=\sqrt{\left(k_{x}+\frac{m \pi}{d}\right)^{2}+\left(k_{y}+\frac{n \pi}{d}\right)^{2}} .
$$

Typical dispersion relationships are shown in fig. 2 for the mechanical parameters reported in [43] and corresponding to:

$$
\begin{array}{lll}
\rho=10^{5} \mathrm{~kg} / \mathrm{m}^{3}, & \lambda=2.8 \times 10^{10} \mathrm{~N} / \mathrm{m}^{2}, & \mu=4 \times 10^{9} \mathrm{~N} / \mathrm{m}^{2}, \\
J=10^{4} \mathrm{~kg} / \mathrm{m}, & \eta+\varepsilon=1.62 \times 10^{9} \mathrm{~N}, & \alpha=2 \times 10^{9} \mathrm{~N} / \mathrm{m}^{2} .
\end{array}
$$


The figure 2 shows the cut-off frequency associated with the microrotational wave, together with the limit cases for the phase and group speeds.
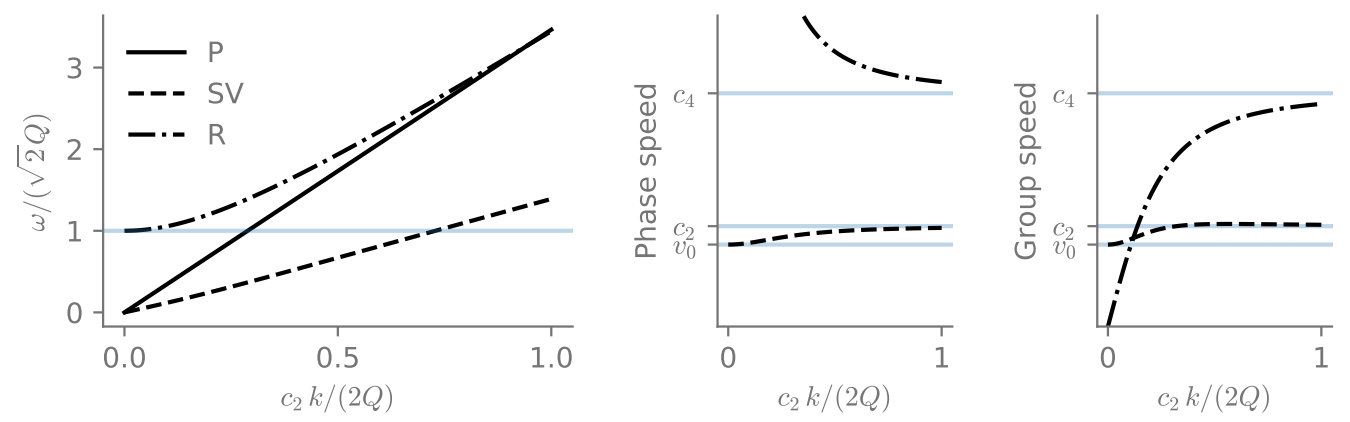

Figure 2. Dispersion relations for a homogeneous micro-polar material material with properties: $\rho=$ $1 \times 10^{5}, J=1 \times 10^{4}, \lambda=2.8 \times 10^{10}, \eta+\varepsilon=1.62 \times 10^{9}, \mu=4 \times 10^{9}, \alpha=2 \times 10^{9}$ as in [43]. The plot on the left shows the frequency-wave number relation for the non-dispersive P-wave (continuous line) and the dispersive SV and micro-rotational wave (dashed lines). The microrotational wave is only triggered above the normalized frequency of 1 . The plots in the middle and right part of the figure show the phase and group speeds for the dispersive modes.

Figure 3 compares now the analytic and numerical band structure for the micro-polar solid for the following set of material parameters:

$$
\begin{array}{lll}
\rho=2770 \mathrm{~kg} / \mathrm{m}^{3}, & \lambda=5.12 \times 10^{10} \mathrm{~N} / \mathrm{m}^{2}, & \mu=2.76 \times 10^{10} \mathrm{~N} / \mathrm{m}^{2}, \\
J=306.5 \mathrm{~kg} / \mathrm{m}, & \eta+\varepsilon=7.66 \times 10^{9} \mathrm{~N}, & \alpha=3.07 \times 10^{9} \mathrm{~N} / \mathrm{m}^{2} .
\end{array}
$$

The numerical curves were obtained with a mesh of $34 \times 34$ bilinear elements. The values for the classical model material parameters are those of aluminum, while the ones for the micropolar model have been adjusted to yield a normalized cut-off frequency of 2 . The figure also shows the unit material cell and the first Brillouin zone. The numerical implementation accurately predicts the propagation modes, including the micro-rotational wave, together with the analytic value of the cut-off frequency. 
Unit cell

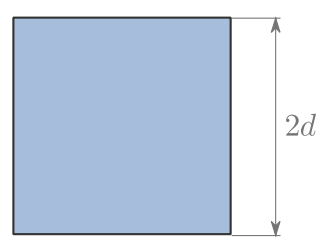

First Brillouin zone

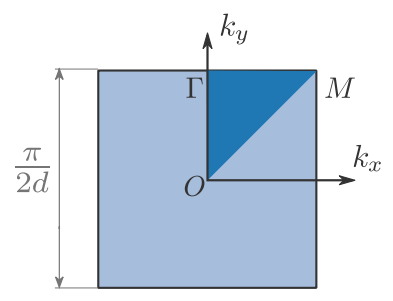

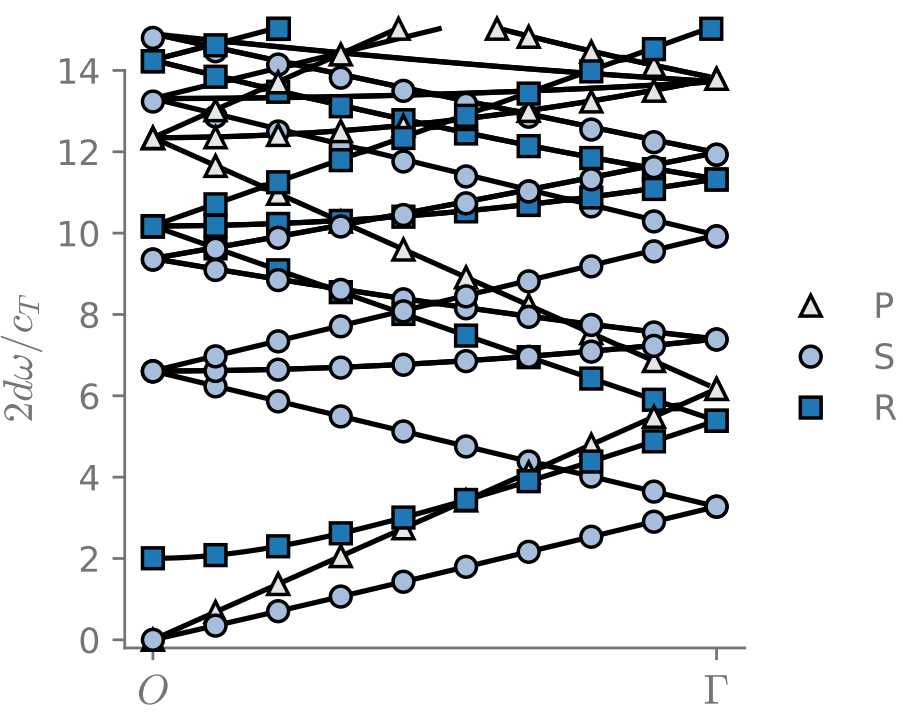

Figure 3. Dispersion relations for a homogeneous micro-polar material model. Solid lines represent FEM results while markers correspond to the analytic solution. The left part of the figure shows the unit material cell and the first Brillouin zone.

As an additional verification, we also tested the convergence in the calculation of the dispersion relations after considering the first 12 modes for a sequence of meshes of $2 \times 2,4 \times 4,8 \times 8$, and $16 \times 16$ elements. The error in the eigenvalue computation was measured according to

$$
e=\frac{\left\|\boldsymbol{\omega}_{\mathrm{ref}}-\boldsymbol{\omega}_{h}\right\|_{2}}{\left\|\boldsymbol{\omega}_{\mathrm{ref}}\right\|_{2}}
$$

where $\boldsymbol{u}_{h}$ is the set of eigenvalues (dispersion relation) for a mesh of characteristic element size $h$ and $\boldsymbol{u}_{\text {ref }}$ is the solution corresponding to the $32 \times 32$ elements mesh, which has been taken as reference. The results for this sequence, together with the variation in the error parameter, are displayed in fig. 4. The estimated convergence rate for the eigenvalues is 1.81 . 

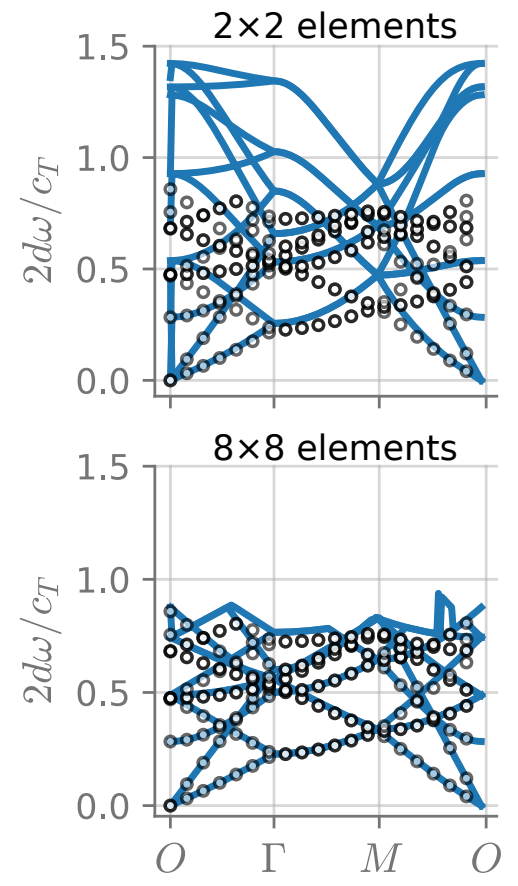
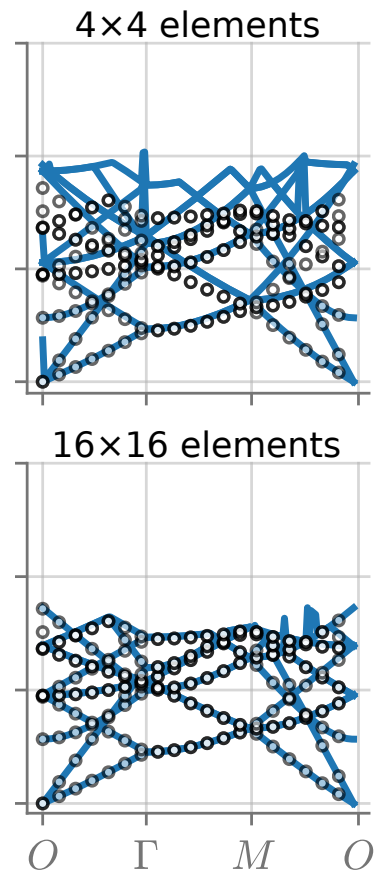

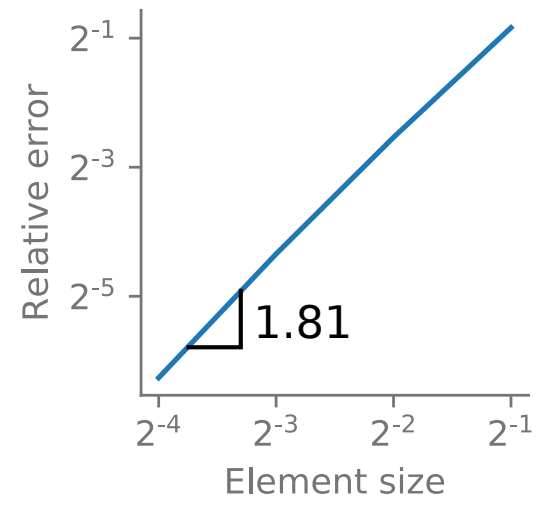

Figure 4. Convergence of the first 12 modes in the dispersion relations for a sequence of meshes with: $2 \times 2,4 \times 4,8 \times 8$, and $16 \times 16$ elements - presented as solid blue lines in the background. The results are compared with a mesh that has $32 \times 32$ elements - presented as dots in the foreground. The estimated convergence rate for the eigenvalues in the 2-norm is 1.81 .

\subsection{Variation of micropolar parameters in a bilayer composite}

We considered a bilayer composite made with two materials that share all the properties, except for one of the micropolar parameters. Thus, we varied $J, \alpha$ and $\xi \equiv \eta+\varepsilon$, while keeping the other parameters fixed. Notice that the composite represents a homogeneous material in the case that both layers share the same material properties. Figure 5 shows the unit cell and the first Brillouin zone for this set of analyses. 


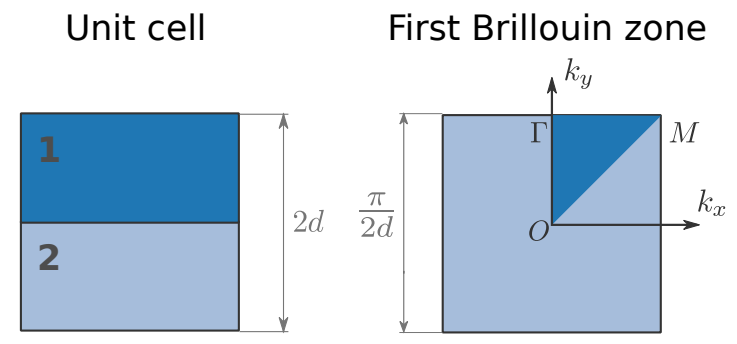

Figure 5. (Left) Schematic of the unit cell for the bilayer material. (Right) Illustration of the first Brillouin zone and the irreducible Brillouin zone.

The properties in material 2, as presented in fig. 5, are fixed. The values used are the following:

$$
\begin{array}{lrl}
\rho_{1} & =\rho_{2}=2770 \mathrm{~kg} / \mathrm{m}^{3}, & \lambda_{1}=\lambda_{2}=5.12 \times 10^{10} \mathrm{~Pa}, \\
\mu_{1}=\mu_{2}=2.76 \times 10^{10} \mathrm{~Pa}, & \alpha_{2}=3.07 \times 10^{9} \mathrm{~Pa}, \\
\xi_{2}=7.66 \times 10^{10} \mathrm{~N}, & J_{2}=306.5 \mathrm{~kg} / \mathrm{m} .
\end{array}
$$

Figure 6 presents the results for variations in $J_{1} \in\{30,100,300,1000,3000\} \mathrm{kg} / \mathrm{m}$. The results are compared with those of the homogeneous cell shown by the black dots, while the dispersion curves resulting from variations in $J_{1}$ are described by the continuous blue line. As $J_{1}$ increases the cut-off frequency for the microrotational wave decreases, which is due to the overall increase in the inertial density. We can also highligh that for larger values of $J_{1}$ the dispersion for the $S V$ and $T R$ waves increases (see the results for $J_{1} / J_{2}=10$ ). This is a result of the interaction between $T R$ and $S V$ waves, but $P$ waves are not affected since they do not interact when the incidence is perpendicular. 

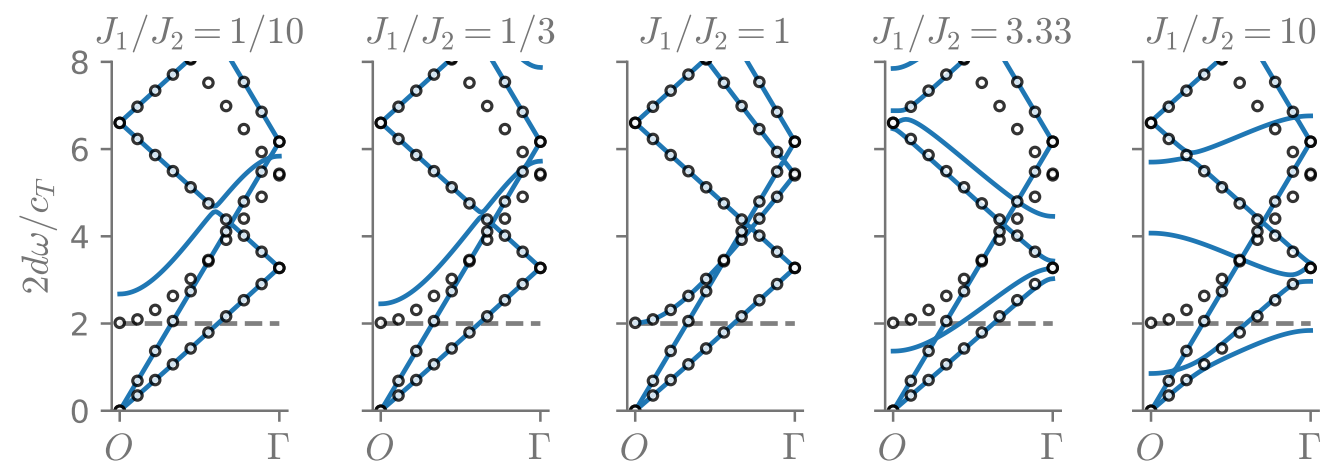

Figure 6. Dispersion curves for a bilayer composite made with two micropolar materials. All material parameters are fixed except for the inertial density $J_{1}$, which takes values in $\{30,100,300,1000,3000\} \mathrm{kg} / \mathrm{m}$. The dispersion curves for the different values of $J_{1}$ correspond to the continuous blue line, while black dots show reference results corresponding to a homogeneous material cell. The increase in $J_{1}$ produces an overall increase in the inertial density accompanied by a decrease in the cut-off frequency of the microrotational wave.

As a second modification to the set of material properties we now changed $\alpha_{1} \in\{3.41 \times$ $\left.10^{8}, 1.02 \times 10^{9}, 3.07 \times 10^{9}, 9.21 \times 10^{9}, 2.76 \times 10^{10}\right\}$ Pa. The corresponding results, in terms of dispersion curves are shown in fig. 7. It is now evident how the cut-off frequency presents an opposite trend as compared with the variations in $J_{1}$, that is, the cut-off frequency increases as the ratio $\alpha_{1} / \alpha_{2}$ increases. This is also an expected result considering the overall increase in $\alpha$ for the composite. Furthermore, we see that we can increase the size of the (partial) bandgap increasing the ratio $\alpha_{1} / \alpha_{2}$. 

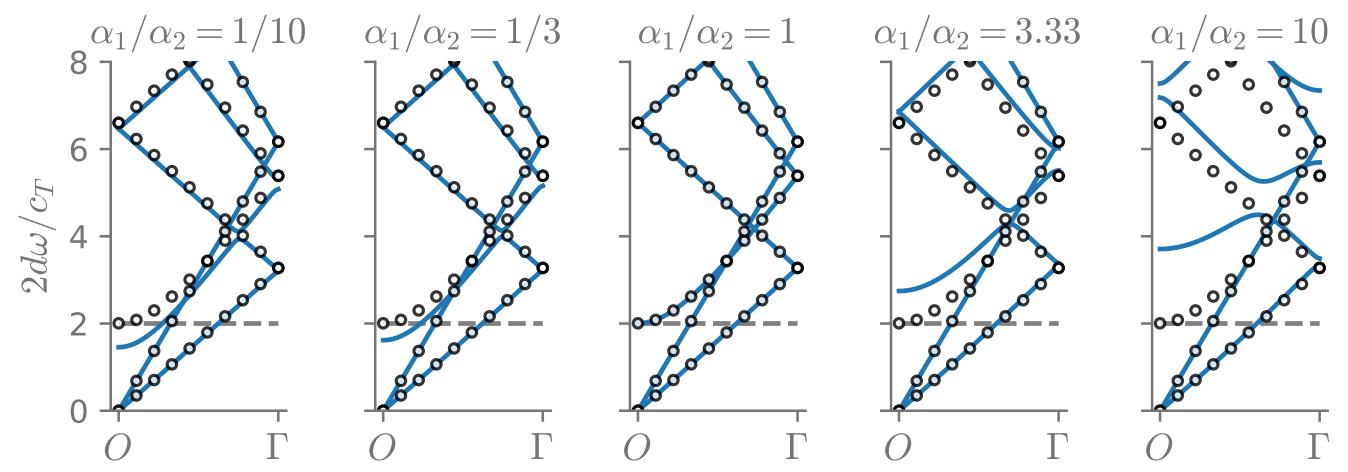

Figure 7. Dispersion curves for a bilayer composite made with two micropolar materials. All material parameters are fixed except for $\alpha_{1}$, which takes values in $\left\{3.41 \times 10^{8}, 1.02 \times 10^{9}, 3.07 \times\right.$ $\left.10^{9}, 9.21 \times 10^{9}, 2.76 \times 10^{10}\right\} \mathrm{Pa}$. The dispersion curves for the different values of $\alpha_{1}$ correspond to the continuous blue line, while the black dots show reference results corresponding to a homogeneous material cell. The overall increase of the $\alpha$ parameter for the composite produces a decrease in the cut-off frequency and an increase in the dispersion for the SV waves.

We considered as a last variation changes in the parameter $\xi_{1}=\eta_{1}+\epsilon_{1} \in\left\{8.51 \times 10^{8}, 2.55 \times\right.$ $\left.10^{9}, 7.66 \times 10^{9}, 2.30 \times 10^{10}, 6.89 \times 10^{10}\right\} \mathrm{N}$. Although this parameter changes the dispersive response, the cut-off frequency for the micro-rotational wave remains unmodified, as $Q$ is independent of $\xi$ and therefore the cut-off frequency is independent of the overall modulus of the composite. Although we see some (partial) bandgaps when changing the ratio $\xi_{1} / \xi_{2}$, it is more interesting to highlight how the hybridization between $S V$ and $T R$ modes changes across the different values of the ratio. 

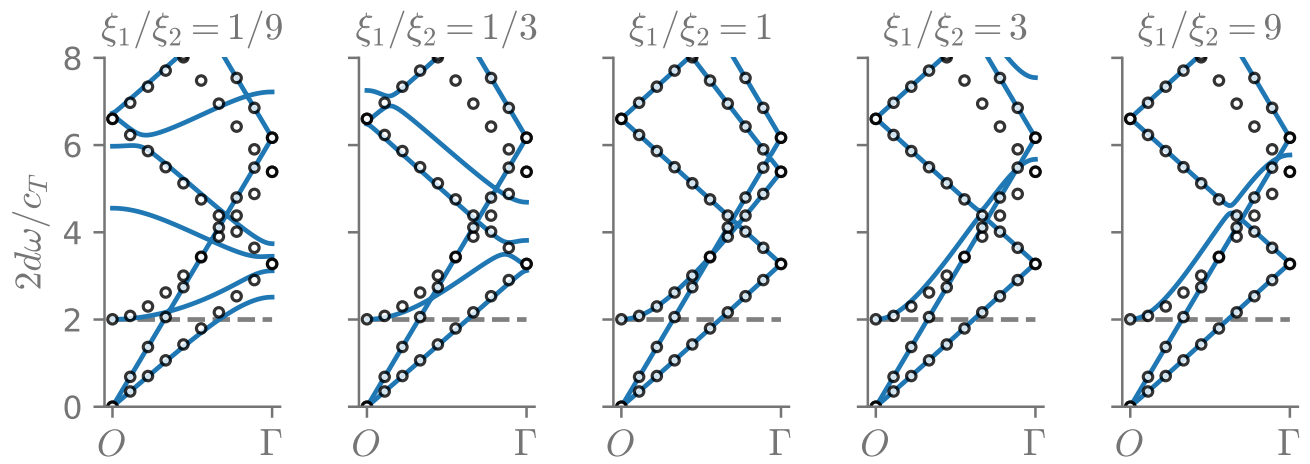

Figure 8. Dispersion curves for a bilayer composite made with two micropolar materials. All material parameters are fixed except for $\xi_{1}$, which takes values in $\left\{8.51 \times 10^{8}, 2.55 \times 10^{9}, 7.66 \times\right.$ $\left.10^{9}, 2.30 \times 10^{10}, 6.89 \times 10^{10}\right\} \mathrm{N}$. The dispersion curves for the different values of $\xi_{1}$ correspond to the continuous blue line, while the black dots show reference results corresponding to a homogeneous material cell. In this case the cut-off frequency remains unmodified as $Q$ is independent of $\xi$.

\subsection{Variation of microstructural length}

Although the micropolar medium introduces dispersive behavior through the presence of length scale parameters, additional frequency dependence of the wave propagation velocity in the medium can be obtained if we explicitly consider the presence of microstructural features embedded in the micropolar medium (fig. 9). Here we explore a material cell which is composed of a circular pore of diameter $d$ embedded inside a micropolar medium.

\section{Unit cell}

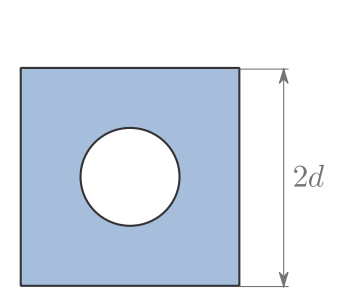

First Brillouin zone

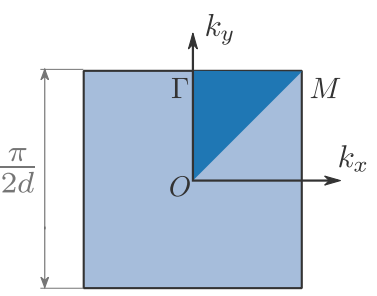

Figure 9. (Left) Schematic of the unit cell for the porous material. (Right) Illustration of the first Brillouin zone and the irreducible Brillouin zone.

We will assume a pore with a diameter that is $1 / 2$ of the cell length or equivalently a porosity 
of $\pi / 16 \approx 0.196$. This value is kept fixed as we modify the values of the unit cell. We will use the following set of mechanical parameters for the micropolar model:

$$
\begin{array}{lr}
\rho=2770 \mathrm{~kg} / \mathrm{m}^{3}, & \lambda=5.12 \times 10^{10} \mathrm{~Pa}, \\
\mu=2.76 \times 10^{10} \mathrm{~Pa}, & \alpha=3.07 \times 10^{9} \mathrm{~Pa}, \\
\eta+\varepsilon=7.66 \times 10^{10} \mathrm{~N}, & J=306.5 \mathrm{~kg} / \mathrm{m} .
\end{array}
$$

For the size effect analysis, it is convenient to express the mechanical parameter in terms of a constitutive length scale present in the micropolar model as discussed in [44] and given by:

$$
\ell^{2} \equiv \frac{\eta+\varepsilon}{2 \mu}=0.3725 \mathrm{~m}
$$

in which $2 \mu \ell^{2}$ represents the rotational stiffness of the material [32]. Notice that a change in the size of the unit cell implies a change in the pore diameter and therefore a change in the ratio between the intrinsic length scale $\ell$ and the characteristic microstructural dimension $d / \ell$. This variation in the dispersion relations with the ratio $d / \ell$ is shown in fig. 10 .
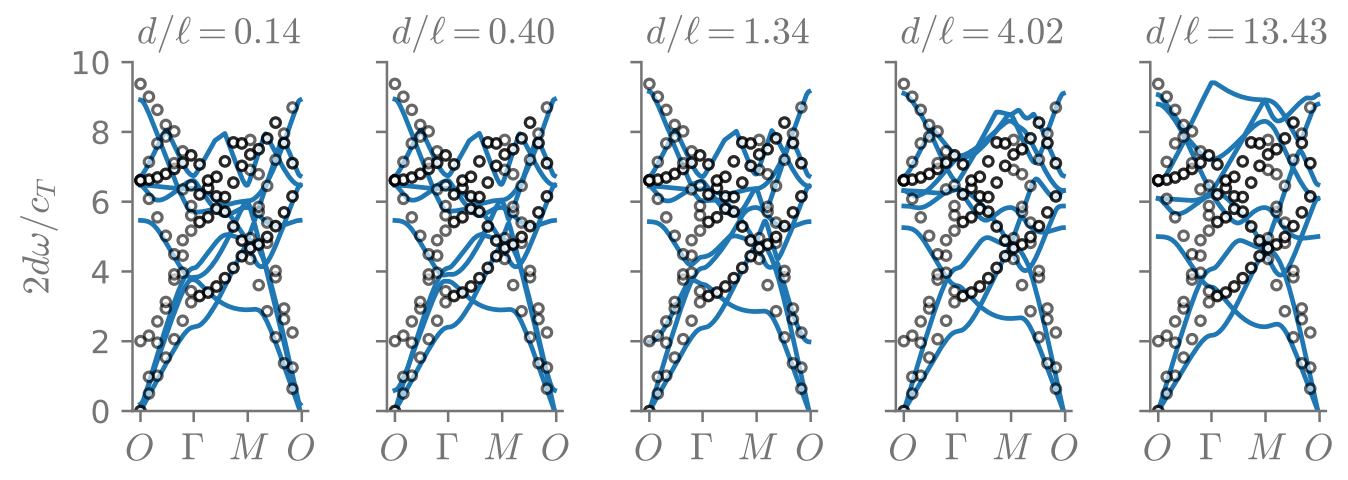

Figure 10. Variation in the band structure for a micropolar periodic cellular material with circular pores for different values of the ratio $d / \ell$.

\subsection{Directionality in cellular material with a circular pore}

As a final result, we computed the directionality curves for cellular materials with circular pores. We changed the diameter of the pore keeping fixed the size of the cell. The material properties used are the same as in Section 4.3. We compare the directionality results in a qualitative fashion, showing how different directions present different phase/group speeds when changing the porosity. 
This comparison is only valid for small wavenumber, because near the edges of the first Brillouin zone the different branches might intersect and the resulting contours would contain information from mixed modes.

Figure 11 presents the directionality curves for the first three branches of the dispersion relations for cellular materials with increasing porosity with values: $0.000,0.196,0.503$ and 0.709 . A porosity of 0 represents a homogeneous material, used as reference in this case. The directionality (anisotropy) of the material increases for higher porosity values.

The first branch presents lower phase speed along the vertical and horizontal directions, while the opposite happens for the second mode. This is expected since these two modes represent quasi-transversal and quasi-longitudinal propagations modes for small wavenumbers. Furthermore, the first mode is the one that presents a higher change in directionality. We can also see that the anisotropy for the third branch is more sensitive to the wavenumber than the porosity, being (almost) isotropic for small wavenumbers. 


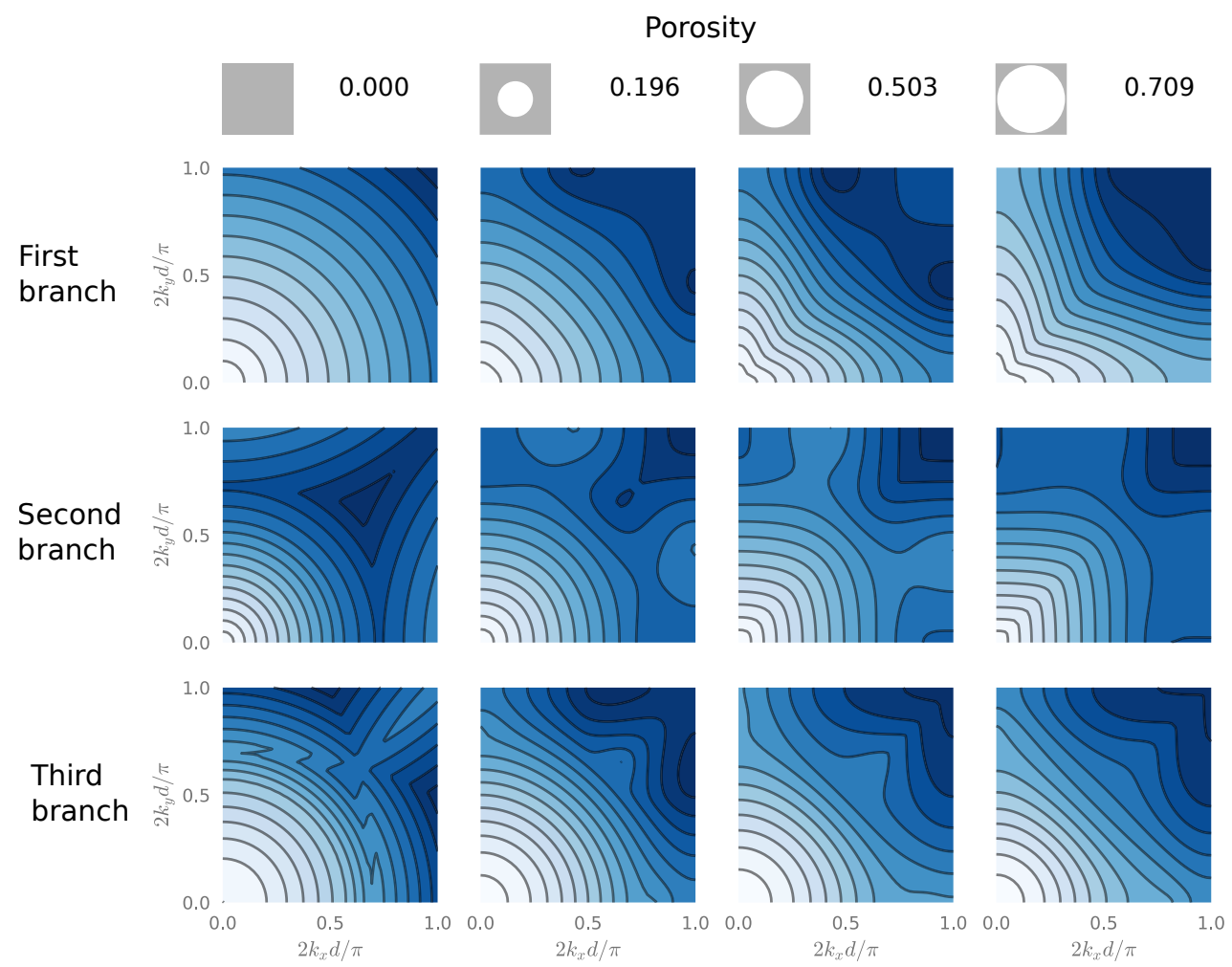

Figure 11. Directionality curves for cellular materials with increasing porosity for the first three branches. The corresponding porosities are: $0.000,0.196,0.503$ and 0.709 . The porosity increases from left to right and is presented at the top. A porosity of 0 represents a homogeneous material, used as reference in this case.

\section{Conclusions}

We discussed several theoretical and simulation aspects related to a Cosserat-like micropolar medium. In particular, we address the model capabilities to capture dispersive behavior through its kinematic assumptions and constitutive parameters. For that purpose, we used the theory of phononic crystals, where the band structure of the material is found from the analysis of FloquetBloch periodicity conditions. Within that context we considered unit material cells corresponding to a homogeneous material, a bilayer composite and a porous cell composed of a circular cavity embedded in a micropolar matrix. The dispersive properties in each case were measured in terms of the variation in the cut-off frequency of the microrotational wave for the different considered 
values of the mechanical and geometric parameters of the model. We found that the ability of the micropolar medium to capture dispersive behavior can be increased mainly through changes in the rotational inertia of the material and the length scale parameter associated to its bending stiffness. Furthermore, these results can serve as a benchmark for a program of physical experiments on periodic solids to explore the existence of microrotational waves, as predicted by micropolar theory.

\section{Appendix A. Terms for the finite element equilibrium equations in the micropolar solid}

The discrete finite element equilibrium equations for the micropolar solid were written as

$$
\left[\begin{array}{cc}
K_{u u}^{k p} & K_{u \theta}^{k p} \\
K_{\theta u}^{k p} & K_{\theta \theta}^{k p}
\end{array}\right]\left\{\begin{array}{l}
u^{p} \\
\theta^{p}
\end{array}\right\}-\omega^{2}\left[\begin{array}{cc}
M_{u u}^{k p} & 0 \\
0 & M_{\theta \theta}^{k p}
\end{array}\right]\left\{\begin{array}{l}
u^{p} \\
\theta^{p}
\end{array}\right\}=\left\{\begin{array}{c}
f_{e x t} \\
q_{\text {ext }}
\end{array}\right\},
$$

where each one of the terms are defined next as follows,

$$
K_{u u}^{k p}=\int_{V} B_{i j}^{k} C_{i j r s} B_{r s}^{p} \mathrm{~d} V+\int_{V} \hat{W}_{i j}^{k} G_{i j r s} \hat{W}_{r s}^{p} \mathrm{~d} V \equiv \int_{V} B_{i j}^{k} C_{i j r s} B_{r s}^{p} \mathrm{~d} V+\int_{V} \mu_{c} \hat{W}_{i j}^{k} \hat{W}_{i j}^{p} \mathrm{~d} V,
$$

which is symmetric.

$$
\begin{aligned}
& K_{u \theta}^{k p}=\int_{V} \hat{W}_{i j}^{k} G_{i j r s} R_{r s}^{p} \mathrm{~d} V \equiv \int_{V} \alpha \hat{W}_{i j}^{k} R_{r s}^{p} \mathrm{~d} V \\
& K_{\theta u}^{k p}=\int_{V} R_{i j}^{k} G_{i j r s} \hat{W}_{r s}^{p} \mathrm{~d} V \equiv \int_{V} \alpha R_{i j}^{k} \hat{W}_{r s}^{p} \mathrm{~d} V,
\end{aligned}
$$

with $K_{u \theta}^{k p}=\left(K_{\theta u}^{k p}\right)^{T}$.

$$
K_{\theta \theta}^{k p}=\int_{V} R_{i j}^{k} G_{i j r s} R_{r s}^{p} \mathrm{~d} V+\int_{V} M_{i j}^{k} D_{i j r s} M_{r s}^{p} \mathrm{~d} V \equiv \int_{V} \alpha R_{i j}^{k} R_{r s}^{p} \mathrm{~d} V+\int_{V} M_{i j}^{k} D_{i j r s} M_{r s}^{p} \mathrm{~d} V,
$$

which is also symmetric.

Similarly, the inertial terms, which are both symmetric, are defined as

$$
\begin{aligned}
M_{u u}^{k p} & =\int_{V} \rho_{{ }_{u}} N_{i}^{k}{ }_{u} N_{i}^{p} \mathrm{~d} V \\
M_{\theta \theta}^{k p} & =\int_{V} J_{\theta} N_{i}^{k}{ }_{\theta} N_{j}^{p} \mathrm{~d} V .
\end{aligned}
$$


Finally, the external force and couple vectors read

$$
\begin{aligned}
& f_{\text {ext }}^{p}=\int_{S}{ }_{u} N_{i}^{p} t_{i} \mathrm{~d} S, \\
& q_{\text {ext }}^{p}=\int_{S}{ }_{\theta} N_{i}^{p} m_{i} \mathrm{~d} S .
\end{aligned}
$$

\section{Appendix B. In-plane equations of motion}

The equations of motion for waves in the plane are the following:

$$
\begin{aligned}
& \frac{\lambda+2 \mu}{\rho}\left[\frac{\partial^{2} u_{x}}{\partial x^{2}}+\frac{\partial^{2} u_{y}}{\partial y \partial x}\right]-\frac{\mu+\alpha}{\rho}\left[\frac{\partial^{2} u_{y}}{\partial y \partial x}-\frac{\partial^{2} u_{x}}{\partial y^{2}}\right]+\frac{2 \alpha}{\rho} \frac{\partial \theta_{z}}{\partial y}=-\omega^{2} u_{x}, \\
& \frac{\lambda+2 \mu}{\rho}\left[\frac{\partial^{2} u_{x}}{\partial y \partial x}+\frac{\partial^{2} u_{y}}{\partial y^{2}}\right]-\frac{\mu+\alpha}{\rho}\left[\frac{\partial^{2} u_{x}}{\partial y \partial x}-\frac{\partial^{2} u_{y}}{\partial x^{2}}\right]-\frac{2 \alpha}{\rho} \frac{\partial \theta_{z}}{\partial y}=-\omega^{2} u_{y}, \\
& \frac{2 \alpha}{J}\left[\frac{\partial u_{y}}{\partial x}-\frac{\partial u_{x}}{\partial y}\right]+\frac{\eta+\varepsilon}{J}\left[\frac{\partial^{2} \theta_{z}}{\partial x^{2}}+\frac{\partial^{2} \theta_{z}}{\partial y^{2}}\right]-\frac{4 \alpha}{J} \theta_{z}=-\omega^{2} \theta_{z},
\end{aligned}
$$

notice that the equations do not involve the parameter $\beta$.

We can write the constitutive equations in extended Voigt's notation as

$$
\left\{\begin{array}{c}
\sigma_{x x} \\
\sigma_{y y} \\
\sigma_{x y} \\
\sigma_{y x} \\
\mu_{z x} \\
\mu_{z y}
\end{array}\right\}=\left[\begin{array}{cccccc}
\lambda+2 \mu & \lambda & 0 & 0 & 0 & 0 \\
\lambda & \lambda+2 \mu & 0 & 0 & 0 & 0 \\
0 & 0 & \mu+\alpha & \mu-\alpha & 0 & 0 \\
0 & 0 & \mu-\alpha & \mu+\alpha & 0 & 0 \\
0 & 0 & 0 & 0 & \eta+\varepsilon & 0 \\
0 & 0 & 0 & 0 & 0 & \eta+\varepsilon
\end{array}\right]\left\{\begin{array}{c}
\gamma_{x x} \\
\gamma_{y y} \\
\gamma_{x y} \\
\gamma_{y x} \\
\kappa_{z x} \\
\kappa_{z y}
\end{array}\right\},
$$

where

$$
\begin{gathered}
\gamma_{x x}=\frac{\partial u_{x}}{\partial x}, \quad \gamma_{y y}=\frac{\partial u_{y}}{\partial y}, \\
\gamma_{x y}=\frac{\partial u_{y}}{\partial x}+\theta_{z}, \quad \gamma_{y x}=\frac{\partial u_{x}}{\partial y}-\theta_{z}, \\
\kappa_{z x}=\frac{\partial \theta_{z}}{\partial x}, \quad \kappa_{z y}=\frac{\partial \theta_{z}}{\partial y} .
\end{gathered}
$$

\section{Acknowledgements}

This work was supported by EAFIT and COLCIENCIAS' Scholarship Program No. 6172. 


\section{References}

\section{References}

[1] Mahmoud I Hussein, Michael J Leamy, and Massimo Ruzzene. Dynamics of phononic materials and structures: Historical origins, recent progress, and future outlook. Applied Mechanics Reviews, 66(4):040802, 2014.

[2] Ankit Srivastava. Elastic metamaterials and dynamic homogenization: a review. International Journal of Smart and Nano Materials, 6(1):41-60, 2015.

[3] Biswajit Banerjee. An introduction to metamaterials and waves in composites. Crc Press, 2011.

[4] Roderic Lakes. Elastic and viscoelastic behavior of chiral materials. International Journal of Mechanical Sciences, 43(7):1579-1589, 2001.

[5] Tobias Frenzel, Muamer Kadic, and Martin Wegener. Three-dimensional mechanical metamaterials with a twist. Science, 358(6366):1072-1074, 2017.

[6] Stefano Gonella and Massimo Ruzzene. Analysis of in-plane wave propagation in hexagonal and re-entrant lattices. Journal of Sound and Vibration, 312:125-139, 2008.

[7] David Schurig, JJ Mock, BJ Justice, Steven A Cummer, JB Pendry, AF Starr, and DR Smith. Metamaterial electromagnetic cloak at microwave frequencies. Science, 314(5801):977-980, 2006.

[8] C. Truesdell and R. Toupin. Principles of Classic Mechanics and Field Theory. Encyclopedia of Physics. Springer-Verlag, 1960.

[9] E.L Aero and E.V Kuvshinski. Fundamental equations of theory of elastic media with rotationally interacting particles. Soviet Physics Solid State, 2:1272-1281, 1961.

[10] R. A. Toupin. Elastic materials with couple-stresses. Archives of Rational Mechanics and Analysis, 11:385-414, 1962.

[11] R. Mindlin and H. Tiersten. Effects of couple-stresses in linear elasticity. Archives of Rational Mechanics and Analysis, 11:415-448, 1962. 
[12] W.T Koiter. Couple stresses in the theory of elasticity. Proc.K.Ned.Akad.Wet(B), 67:17-44, 1964.

[13] R. Mindlin. Micro-structure in linear elasticity. Archives of Rational Mechanics and Analysis, $16: 51-78,1964$.

[14] R. Mindlin. Second gradient of strain and surface tension in linear elasticity. International Journal of Solids and Structures, 1:417-438, 1965.

[15] R. Mindlin and N.N Eshel. On first strain gradient theories in elasticity. International Journal of Solids and Structures, 4:109-124, 1968.

[16] A.C Eringen. Linear theory of micropolar elasticity. Journal of Mathematics and Mechanics, 15:909-923, 1966.

[17] Fan Yang, ACM Chong, David Chuen Chun Lam, and Pin Tong. Couple stress based strain gradient theory for elasticity. International Journal of Solids and Structures, 39(10):2731-2743, 2002.

[18] Ali R Hadjesfandiari and Gary F Dargush. Couple stress theory for solids. International Journal of Solids and Structures, 48(18):2496-2510, 2011.

[19] Ali R Hadjesfandiari, Arezoo Hajesfandiari, and Gary F Dargush. Skew-symmetric couplestress fluid mechanics. Acta Mechanica, 226(3):871-895, 2015.

[20] E. Cosserat and F. Cosserat. Théorie des Corps Déformables. A Hermann et Fils, 1909.

[21] W. Voigt. Lehrbuch der Kristalphysik. Teubner, B, 1910.

[22] Witold Nowacki. Theory of asymmetric elasticity. Pergamon Press, Headington Hill Hall, Oxford OX 30 BW, UK, 1986., 1986.

[23] William D Nix and Huajian Gao. Indentation size effects in crystalline materials: a law for strain gradient plasticity. Journal of the Mechanics and Physics of Solids, 46(3):411-425, 1998.

[24] NA Fleck and JW Hutchinson. Strain gradient plasticity. Advances in applied mechanics, 33: 296-361, 1997. 
[25] NA Fleck, GM Muller, MF Ashby, and JW Hutchinson. Strain gradient plasticity: theory and experiment. Acta Metallurgica et Materialia, 42(2):475-487, 1994.

[26] J S Stolken and AG Evans. A microbend test method for measuring the plasticity length scale. Acta Materialia, 46(14):5109-5115, 1998.

[27] M. Takeo. Ground rotational motions recorder in near-source region of earthquakes. Geophysical Research Letters, 25:789-792, 1998.

[28] M. Takeo. Ground rotational motions recorder of earthquakes. Geophysical Research Letters., Vol 26:789-792, 1998.

[29] M. Takeo and H. Ito. What can be learned from rotational motions excited by earthquakes? International journal of Geophyisics, 129:319-329, 1997.

[30] G. Midya. On Love-type surface waves in homogeneous micropolar elastic media. International Journal of Engineering Science, 42:1275-1288, 2004.

[31] A. Merkel, Vincent Tournat, and V. Gusev. Experimental evidence of rotational elastic waves in granular phononic crystals. Physical Review Letters, 107(22):225502, 2011.

[32] Patrizia Trovalusci, Martin Ostoja-Starzewski, Maria Laura De Bellis, and Agnese Murrali. Scale-dependent homogenization of random composites as micropolar continua. European Journal of Mechanics-A/Solids, 49:396-407, 2015.

[33] Sokratis N Iliopoulos, Dimitrios G Aggelis, and Demosthenes Polyzos. Wave dispersion in fresh and hardened concrete through the prism of gradient elasticity. International Journal of Solids and Structures, 78:149-159, 2016.

[34] Sokratis N Iliopoulos, Fabian Malm, Christian U Grosse, Dimitrios G Aggelis, and Demosthenes Polyzos. Concrete wave dispersion interpretation through Mindlin's strain gradient elastic theory. The Journal of the Acoustical Society of America, 142(1):EL89-EL94, 2017.

[35] G. Thomas Mase, Ronald E. Smelser, and George E. Mase. Continuum mechanics for engineers. CRC press, 2009. 
[36] R. Lakes. Experimental micro mechanics methods for conventional and negative Poisson's ratio cellular solids as Cosserat continua. Journal of Engineering Materials and Technology, 113(1): 148-155, 1991.

[37] Soroosh Hassanpour and Glenn R Heppler. Micropolar elasticity theory: a survey of linear isotropic equations, representative notations, and experimental investigations. Mathematics and Mechanics of Solids, 22(2):224-242, 2017.

[38] Soroosh Hassanpour and G. R. Heppler. Step-by-step simplification of the micropolar elasticity theory to the couple-stress and classical elasticity theories. In ASME 2014 International Mechanical Engineering Congress and Exposition, pages V009T12A042-V009T12A042. American Society of Mechanical Engineers, 2014.

[39] Leon Brillouin. Wave propagation in periodic structures: Electric filters and crystal lattices. Dover Publications, 1 edition, 1953.

[40] Charles Kittel. Introduction to Solid State Physics. Wiley, 7 edition, 1996.

[41] Stephen C Cowin. An incorrect inequality in micropolar elasticity theory. Zeitschrift für Angewandte Mathematik und Physik (ZAMP), 21(3):494-497, 1970.

[42] Nicolás Guarín-Zapata and Juan Gomez. Evaluation of the spectral finite element method with the theory of phononic crystals. Journal of Computational Acoustics, 23(02):1550004, 2015.

[43] M. Kulesh. Waves in linear elastic media with microrotations, part 1: Isotropic full cosserat model. Bulletin of the Seismological Society of America, 99(2B):1416-1422, 2009.

[44] S. C. Cowin. Stress functions for Cosserat elasticity. International Journal of Solids and Structures, 6(4):389-398, 1970. 